ISSN 1816-6075 (Print), 1818-0523 (Online)

Journal of System and Management Sciences

Vol. 10 (2020) No. 1, pp. 32-50

DOI:10.33168/JSMS.2020.0102

\title{
Design of Tour Guide Outsourcing Service Contract under Dual Principal-Agent
}

\author{
Fang $\mathrm{Lu}^{1}$, Jiangshun $\mathrm{Zhang}^{2}$ and Jijun $\mathrm{Yu}^{2}$ \\ ${ }^{1}$ School of Logistics and Transportation, Central South University of Forestry and \\ Technology \\ ${ }^{2}$ School of Arts and Communication, China University of Geosciences (Wuhan)
}

Corresponding Author: 717290412@qq.com

\begin{abstract}
The tourism supply chain provides intangible service products. The tourists who participate in the whole service process determine the quality of tourism service. Presently, there is still no effective incentive mechanism for the members involved in tourism service outsourcing, which leads to the increasingly intensified contradiction among travel agencies, guide service providers and tourists. Based on the dual principal-agent status of travel agencies, this paper constructs a tour guide outsourcing cooperative service contract model among tourists, travel agencies and guide service providers, and analyzes the importance of rationally setting performance sharing mechanism and reward and punishment mechanism for travel agencies to improve the satisfaction of tourists effectively. To resolve the conflicts among tourists, travel agencies and tour guides in the existing tourism service outsourcing, this paper proposes the issue of adding reward and punishment mechanisms based on the performance sharing mechanism to improve the effectiveness of tourists.
\end{abstract}

Keywords: Service outsourcing, dual principal-agent, service quality

\section{Introduction}

Travel agencies have dual identities in tourism activities, both as agents and principals. Tourists entrust travel agencies to arrange travel activities throughout the journey. The travel agency entrusts tour guides to serve tourists throughout the journey. Tourists evaluate the service products of travel agencies through tour guide services. Tour guides are an important link between tourists and travel agencies. They arrange food, accommodation, travel, sightseeing, shopping, entertainment and other activities for tourists, and the key role in the entire tourism supply chain is beyond doubt. The quality of tour guide services directly affects the satisfaction of 
tourists and even the health and sustainable development of the entire tourism industry.

Guide service outsourcing is an important part of tourism service outsourcing. In recent years, disputes among tourists, travel agencies and tour guides have been increasing. Tourists have maintained a high complaint rate for the service quality of tour guide and have been increasing year by year. Most of the existing contract designs are for the product supply chain, but the intangibility of the products provided by the tourism supply chain and the "logistics" with the "passenger flow" as the core make the contract of the existing tourism supply chain. The design of product supply chain is difficult to apply to service supply chain. It is urgent to design a service contract with guide service outsourcing under the dual principalagent relationship to improve the utility of tourists, overcome the contradiction between tourists and travel agencies and tour guides, and improve the quality of tourism services.

\section{Literature Review}

Regarding the quality of service, the academic community generally agrees that consumers themselves determine the quality of service by comparing the "expectation service" and the "perception service" they feel; the quality of service is either subjective or objective quality of service (Gronroos, 1982).

With the discussion of the evaluation methods of tourism service quality by Fick and Ritchie (1991) in 1991, a large number of studies on the quality of tourism services began to appear. Marcjanna and Samuel (1998) measured the satisfaction of tourists by using the SERVQUAL model and used the gap analysis method to study how to improve the quality of tourism services. According to Atilgan (2003), it is more effective to use the corresponding analysis method to assess the satisfaction of service quality of different consumer groups. Travel agencies should consider cultural differences when evaluating tourist satisfaction. Graham (2004) compares the four methods of measuring the quality of tourism services (SERVQUAL, weighted SERVQUAL, IPA and SERVPERF) in tourism enterprises, and concludes that the SERVPERF method is most effective under the condition of limited cost and time. Gina (2014) divides the quality of tourism services into two parts: tourism service process and tourism service level, and based on this, a qualitative evaluation framework for tourism service quality is proposed. Flemming (2015) pointed out that the quality of tourism services is out of the existing tourist experience, so the quality of tourism services is individual. Min (2011) explores the relationship among three elements of tourism service quality: system quality, information quality and service quality.

Sushila (2014) used empirical methods to investigate the tourist satisfaction of rural tourism in Malaysia and gave recommendations for rural tourism. Some scholars have also studied the factors affecting the quality of tourism services in a 
specific region. For example, Athena $(2010,2011)$ believes that tour guides are the most obvious and important factor in ensuring service quality in tourism, and should minimize some of the realities such as unfair measures and systems for tour guides.

Compared with the research on IT service outsourcing, the research on tourism service outsourcing started late, and its research content and theory stayed in macro decision-making. Based on summarizing the elements restructuring and service outsourcing services value chain theory, Fan Wei (2012) preliminarily defined the connotation of tourism service outsourcing, that is, tourism service outsourcing is the process reengineering of the current tourism economic industry, it can solve the current situation of "small, weak and poor" in the tourism service industry to some extent. Tourism service outsourcing is an important way to upgrade the tourism industry and optimize tourism resources.

Tourism service outsourcing focuses on two aspects. First, the description of the overall development of tourism service outsourcing (Lamminmaki, 2008), discusses the feasibility and necessity of service outsourcing, outsourcing management and development model, path, etc. Second, the specialized research on service outsourcing (Chatzoglou, 2009) focuses on the analysis of outsourcing status, motivation and performance of travel agencies, restaurants, and human resources in tourist attractions. Research hotspots on tourism service outsourcing focused on hotel business outsourcing: Espino-Rodríguez (2004) believes that hotel service outsourcing will have a significant positive impact on hotel performance, not only reducing hotel operating costs, but also providing consumers with high Quality service. Lamminmaki (2008) found that hotel service quality is an important factor affecting the complexity of hotel accounting outsourcing when studying the outsourcing decision of hotel accounting system.

Lamminmaki (2011) proposed that the hotel service industry is suitable for the strategy of service outsourcing due to the uncertainty of demand and the large demand for labor. However, Hiamey (2013) pointed out that the core business of the hotel cannot be outsourced, and that outsourcing of services does not always lead to positive results. Leeman (2012), Donada (2009) believes that during the outsourcing of hotel services, maintaining good communication, tolerance and trust, and positive emotions between hotels and service providers is crucial for maintaining production, building lasting partnerships and corporate performance.

Principal-Agent Theory refers to the selection of a subject by one or more subjects through formal or informal agreement, by granting decision-making power to provide services and giving corresponding rewards (Delreu, 2017). First proposed by Holmstrom (1991), the multi-task principal-agent theory applied to service outsourcing continuously. Susarla (2010) used empirical methods to explore the influence of factors such as observability of tasks under multitasking agents in the SaaS model on contract selection. Kristensen (2016) studied ways to promote 
hospital health care performance under multi-tasking goals. Rud (2017) studied the dual principal-agent relationship among commercial banks, financial managers and fund managers in the financial market, and found that intermediaries can protect customers' benefits more than intermediary competition.

The existing literature on tourism service outsourcing mainly focuses on the introduction and definition of the basic concepts and framework of tourism service outsourcing. The research content is mostly at the theoretical and conceptual level, and there is no discussion and analysis of various behaviors and strategies of tourism service outsourcing members. The characteristics of tourism services determine that travel agencies play an important role in the entire service outsourcing system. The dual identity of the agency determines the particularity and complexity of the travel service outsourcing contract design with the travel agency as the core. Presently, there are still few studies on dual principal agent on tourism service outsourcing yet.

\section{Description of the Problems}

This paper discusses the guide service outsourcing system consisting of travel agencies, tour guides and tourists. The output of the outsourcing system determined by the cooperative services of travel agencies and tour guides, and the effectiveness of visitors depends on the level of effort of both. Figure 1 shows the principal-agent relationship among the travel agency, guide service provider and tourists. Among them, the travel agency has the dual identities of the principal and the agent.

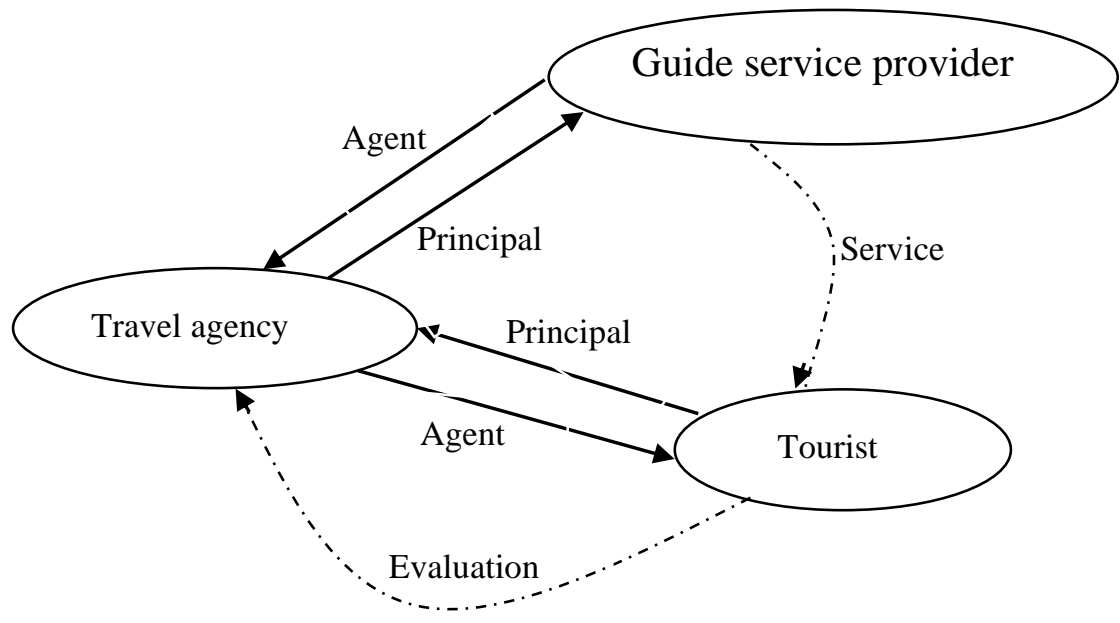

Fig. 1: Tour Guide service outsourcing.

Based on the principal-agent relationship among the tourists-travel agencyguide service provider, assumed that the travel agency (recorded as A) is risk 
neutral, the guide service provider (recorded as G) is risk aversion, and the tourist (recorded as T) is risk neutral. Travel agencies and tour guides provide services to tourists at the same time. They share the benefits of serving tourists and work together to maximize the effectiveness of tourists. The problem studied in this paper is about travel agency how to design an effective incentive contract to maximize the utility of tourists under the dual agent role. The following are basic assumptions and parameter descriptions.

(1) Travel agencies and guide service providers participate in the outsourcing of tour guide services, and work together to maximize the effectiveness of tourists. The cost function of the travel agency is $C_{A}\left(e_{A}, k_{A}\right)=c_{A} e_{A}^{2} / 2 k_{A}, e_{A}$ is the level of effort of the travel agency, $k_{A}$ and $c_{A}$ is the service capacity and effort cost factor of the travel agency. Similarly, $C_{G}\left(e_{G}, k_{G}\right)=c_{G} e_{G}^{2} / 2 k_{G}$ is the cost function of the guide service provider, where $e_{G} 、 k_{G}$ and $c_{G}$ is the effort level, service capability and effort cost coefficient of the guide service provider respectively. The effort cost function of the travel agency and the tour guide service provider satisfies $\partial C_{A}\left(e_{A}, k_{A}\right) / \partial e_{A}>0 \quad, \quad \partial^{2} C_{A}\left(e_{A}, k_{A}\right) / \partial e_{A}^{2}>0 \quad, \quad \partial C_{G}\left(e_{G}, k_{G}\right) / \partial e_{G}>0 \quad$ and $\partial^{2} C_{G}\left(e_{G}, k_{G}\right) / \partial e_{G}^{2}>0$, that is, the law of increasing cost and marginal increase, the economic significance is that the effort cost of the travel agency and the guide service provider increases and speeds with the increase of the effort level.

(2) The guide service has the characteristics of intangibility and perishability, which leads to the hard work of travel agencies and guide service providers during the execution of the outsourcing contract, and the service results are difficult to measure accurately. This paper builds system output based on the total number of tourists received by travel agencies and tour guides, and the total amount of visitor reception is positively related to the service capacity and effort of the tour guide. Therefore, the total number of visitors received during the cycle of the tour guide service outsourcing system is $q=k_{A} e_{A}+k_{G} e_{G}+\varepsilon$, where $e_{A}\left(e_{G}\right)$ is the level of effort of the travel agency (guide service provider), and $k_{A}\left(k_{G}\right)$ is the service capacity of the travel agency (guide service provider). $\varepsilon \square N\left(0, \sigma^{2}\right), \varepsilon$ is the influence of uncertain factors in the tourism market on the performance of tour guide service providers, $\sigma^{2}$ indicating the degree of uncertainty in the tourism market. Therefore, the output of the tour guide service outsourcing system can be $Q=\tau q=\tau\left(k_{A} e_{A}+k_{G} e_{G}+\varepsilon\right), \tau(>0)$ is the net average income obtained by the travel agency from the unit tourists, which is essentially the average price of all the travel products purchased by the travel agency for the tourists.

(3) Under the bilateral moral hazard, the customer and the service provider as a whole need to share the risk, so the output in the service outsourcing contract must achieve the effect of sharing the risk through benefit sharing. In this paper, the travel agency pays the tour guide servicer in a way that uses the performance sharing mechanism, that is, the payment function is $\alpha+\beta Q$, the sum of $\alpha$ and $\beta$ is 
the fixed salary and performance sharing coefficient (also known as the incentive intensity coefficient) of the guide service provider respectively, $\alpha>0,0<\beta<1$.

(4) Description of other parameters: $\Pi_{A}$ is the expected return of the travel agency; $\Pi_{G}$ is the expected return of the guide service provider; $\Pi_{G}^{0}$ for the reserve income of the guide service provider, if the expected benefit $\Pi_{G}$ of the guide service provider is lower than the value, refuse the contract.

The tourist considered in this paper refers to a group of tourists who purchase travel agency tourism products in a service cycle rather than a single individual. The utility of the tourists consists of the basic utility of the package tour $v$ ( $v$ for all visitors (Haiyang, 2009)), the tourist experience of the tourists $Y_{T}$ and the cost of purchasing the tourism products $C_{T}$ (the package price). The tourist experience of a visitor $Y_{T}$ depends on the service capabilities and level of effort of the travel agency and guide service provider, which is $Y_{T}=\theta\left(k_{A} e_{A}+k_{G} e_{G}\right)$. Among them, the constant coefficient $\theta$ indicates the level of preference of visitors. Since the tourists studied in this paper refer to the group of tourists, the cost of tourists purchasing tourism products $C_{T}$ is equal to the product of the total number of tourists $q$ and the net average income of travel agencies from the unit visitors $\tau$, which can be $C_{T}=\tau q=\tau\left(k_{A} e_{A}+k_{G} e_{G}\right)$. In summary, the tourist utility function is equal to the difference between the tourist experience of the visitor and the purchase cost of the visitor. The functional relationship is as follows:

$$
\Pi_{T}=Y_{T}-C_{T}=v+\theta\left(k_{A} e_{A}+k_{G} e_{G}\right)-\tau\left(k_{A} e_{A}+k_{G} e_{G}\right)
$$

\section{Design of Tour Guide Service Outsourcing Contract}

The reward and punishment mechanism is an incentive mechanism for travel agency providers to protect the effectiveness of tourists. The outsourcing contract designed in this paper is two types: reward and punishment mechanism and no reward and punishment mechanism.

\subsection{Design of tour guide service outsourcing contract under no reward and punishment mechanism}

The actual monetary income of the guide service provider consists of two parts: linear payment and effort cost. The impact of uncertainties in the tourism service market will inevitably bring risk costs to tour guide service providers. According to the Arrow-Pratt conclusion, the risk cost record as $\rho \beta^{2} \sigma^{2} / 2$. Among them, $\rho(>0)$ is the risk aversion coefficient of the guide service provider, and the larger the $\rho$, the stronger the risk avoidance of the guide service provider. Since the guide service provider is risk averse, its deterministic equivalent return is equal to the risk cost minus the actual monetary income, that is, the deterministic equivalent return of the guide service provider expressed as: 


$$
\Pi_{G}=\alpha+\beta\left(k_{A} e_{A}+k_{G} e_{G}\right)-\frac{c_{G} e_{G}^{2}}{2 k_{G}}-\frac{1}{2} \rho \beta^{2} \sigma^{2}
$$

Accordingly, the expected return of the travel agency expressed as:

$$
\Pi_{A}=(1-\beta)\left(k_{A} e_{A}+k_{G} e_{G}\right)-\frac{c_{A} e_{A}^{2}}{2 k_{A}}-\alpha
$$

However, while considering the maximization of benefits, travel agencies are also subject to multiple constraints by tour guide providers and their own personal rational constraints (IR) and incentive compatibility constraints (IC). At this time, we can describe the principal-agent relationship between the travel agency and the guide service provider as the following linear programming model P1.

$$
\begin{aligned}
& P 1: \max _{e_{G}, e_{A}} \Pi_{T}=v+\theta\left(k_{A} e_{A}+k_{G} e_{G}\right)-\tau\left(k_{A} e_{A}+k_{G} e_{G}\right) \\
& \text { s.t. }\left(I C_{G}\right) e_{G} \in \arg \max \alpha+\beta \tau\left(k_{A} e_{A}+k_{G} e_{G}\right)-\frac{c_{G} e_{G}^{2}}{2 k_{G}}-\frac{1}{2} \rho \beta^{2} \sigma^{2} \\
& \left(I C_{A}\right) e_{A} \in \arg \max (1-\beta) \tau\left(k_{A} e_{A}+k_{G} e_{G}\right)-\frac{c_{A} e_{A}^{2}}{2 k_{A}}-\alpha \\
& \left(I R_{G}\right) \alpha+\beta \tau\left(k_{A} e_{A}+k_{G} e_{G}\right)-\frac{c_{G} e_{G}^{2}}{2 k_{G}}-\frac{1}{2} \rho \beta^{2} \sigma^{2} \geq \Pi_{G}^{o} \\
& \left(I R_{A}\right)(1-\beta) \tau\left(k_{A} e_{A}+k_{G} e_{G}\right)-\frac{c_{A} e_{A}^{2}}{2 k_{A}}-\alpha \geq \Pi_{A}^{o}
\end{aligned}
$$

Based on linear programming model P1, the optimal effort level of the guide service provider and travel agency under the non-reward and punishment mechanism is as follows:

$$
\begin{aligned}
& e_{G}^{*}=\beta \tau k_{G}^{2} / c_{G} \\
& e_{A}^{*}=(1-\beta) \tau k_{A}^{2} / c_{A}
\end{aligned}
$$

The optimal performance sharing coefficient factor for the above incentive model is:

$$
\beta^{*}=\frac{c_{A} \tau^{2} k_{G}^{3}}{c_{A} \tau^{2} k_{G}^{3}+c_{G} \tau^{2} k_{A}^{3}+c_{A} c_{G} \rho \sigma^{2}}
$$

\subsection{Design of incentive contract for tour guide service outsourcing under the reward and punishment mechanism}

As a contractor and contract designer for the outsourcing of tour guide services, travel agencies are in a dominant position in the process of outsourcing contract execution. It is not easy to observe travel agencies' participation behavior and guide service providers' efforts by each other. In addition, because the output of guide 
service outsourcing is difficult to measure, guide service providers may have a "free rider" problem. To this end, the travel agency can judge the guide service provider's service performance in the form of issuing and recycling questionnaires after the tourist's travel behavior is over, and set a reward and punishment mechanism for its service performance. The service performance measure form of the guide service provider is set to $x=e_{G}+\xi_{G}$. Among them, $e_{G}$ indicates the actual service performance of the guide service provider, $\xi_{G}$ indicating the uncertainty of the service performance observation of the travel agency guide service provider. $\xi_{G} \square N\left(0, \delta^{2}\right)$, where $\delta^{2}$ is the observed error in service performance. This paper sets the reward and punishment mechanism for the service performance of the guide service provider, and makes the reward and punishment intensity coefficient as $Z_{G}=\gamma\left(X_{G}-s_{0}\right)=\gamma\left(e_{G}+\xi_{G}-s_{0}\right), \gamma(\gamma>0)$ is the reward and punishment intensity coefficient, $s_{\mathrm{o}}$ is the service performance standard value set by the travel agency. When $e_{G}+\xi_{G}-s_{0}>0$, the travel agency will give the tour guide service performance reward; when $e_{G}+\xi_{G}-s_{0}<0$, the tour guide service provider will be punished for its service performance; when $e_{G}+\xi_{G}-s_{0}=0$, the tour guide service provider just meets the service performance standard, and the travel agency will not reward the tour guide service provider nor punish it.

At this point, we can describe the principal-agent relationship between the travel agency and the guide service provider as the following linear programming model P2:

$$
\begin{aligned}
& \begin{array}{l}
P 2: \max _{e_{G}, e_{A}} \Pi_{T}=v+\theta\left(k_{A} e_{A}+k_{G} e_{G}\right)-\tau\left(k_{A} e_{A}+k_{G} e_{G}\right) \\
\text { s.t. }\left(I C_{G}\right) e_{G} \in \arg \max \alpha+\beta \tau\left(k_{A} e_{A}+k_{G} e_{G}\right)+\gamma\left(e_{G}-s_{0}\right)-\frac{c_{G} e_{G}^{2}}{2 k_{G}} \\
\quad-\frac{1}{2} \rho \beta^{2} \sigma^{2}-\frac{1}{2} \rho \gamma^{2} \delta^{2} \\
\left(I C_{A}\right) e_{A} \in \arg \max (1-\beta) \tau\left(k_{A} e_{A}+k_{G} e_{G}\right)-\frac{c_{A} e_{A}^{2}}{2 k_{A}}-\gamma\left(e_{G}-s_{0}\right)-\alpha \\
\left(I R_{G}\right) \alpha+\beta \tau\left(k_{A} e_{A}+k_{G} e_{G}\right)-\frac{c_{G} e_{G}^{2}}{2 k_{G}}-+\gamma\left(e_{G}-s_{0}\right)-\frac{c_{G} e_{G}^{2}}{2 k_{G}}-\frac{1}{2} \rho \beta^{2} \sigma^{2} \\
-\frac{1}{2} \rho \gamma^{2} \delta^{2} \geq \Pi_{G}^{o} \\
\left(I R_{A}\right)(1-\beta) \tau\left(k_{A} e_{A}+k_{G} e_{G}\right)-\frac{c_{A} e_{A}^{2}}{2 k_{A}}-\gamma\left(e_{G}-s_{0}\right)-\alpha \geq \Pi_{A}^{o}
\end{array}
\end{aligned}
$$

Based on the linear programming model P2, we can obtain the optimal effort level of guide service providers and travel agencies under the reward and punishment mechanism:

$$
e_{G}^{* *}=\left(\beta \tau k_{G}^{2}+\gamma k_{G}\right) / c_{G}
$$




$$
e_{A}^{* * *}=(1-\beta) \tau k_{A}^{2} / c_{A}
$$

The optimal performance sharing/reward and punishment intensity factors of the above incentive model are:

$$
\begin{aligned}
\beta^{* *} & =\frac{c_{A} \tau^{2} k_{G}^{3}-c_{A} \gamma \tau k_{G}^{2}}{c_{A} \tau^{2} k_{G}^{3}+c_{G} \tau^{2} k_{A}^{3}+c_{A} c_{G} \rho \sigma^{2}} \\
\gamma^{* *} & =\frac{(1-\beta) \tau k_{G}^{2}}{k_{G}+c_{G} \rho \delta^{2}}
\end{aligned}
$$

\subsection{Analysis of two contract designs}

(1)Analysis of incentive and punishment intensity coefficient

Theorem 1: The intensity coefficient of reward and punishment decreases with the increase of performance sharing coefficient, the observation error of the travel agency to the guide service provider, and increases with the increase of the degree of external uncertainty in the tourism market and the average pricing of tourism products.

Proof: Based on equation (20), the partial derivative of $\beta$ is obtained for $\gamma^{* *}$ as $\partial \gamma^{* *} / \partial \beta<0$, that is, the reward and punishment intensity coefficient is a decreasing function with respect to $\beta$. For $\gamma^{* *}$ to find the partial derivative of $\delta^{2}$ as $\partial \gamma^{* *} / \partial \delta^{2}<0$, the greater the observation error of the travel agency to the tour guide service provider, the corresponding reduction of reward and punishment intensity. The simultaneous awards (19) and (20) can further describe the reward and punishment intensity factor as:

$$
\gamma^{* *}=\frac{\tau^{3} k_{G}^{2} k_{A}^{3}+c_{A} \tau k_{G}^{2} \rho \sigma^{2}}{\tau^{2} k_{G} k_{A}^{3}+c_{A} k_{G} \rho \sigma^{2}+\rho \delta^{2}\left(c_{A} \tau^{2} k_{G}^{3}+c_{G} \tau^{2} k_{A}^{3}+c_{A} c_{G} \rho \sigma^{2}\right)}
$$

According to formula (21), the $\gamma^{* *}$ about $\sigma^{2}, \rho$ and $\tau$ partial derivatives are obtained in turn. $\partial \gamma^{* *} / \partial \sigma^{2}>0$, the reward and punishment intensity coefficient is an increasing function of the degree of external uncertainty in the tourism market; $\partial \gamma^{* *} / \partial \rho<0$, the higher the risk avoidance degree of the tour guide service provider, the lower the reward and punishment intensity coefficient is; $\partial \gamma^{* *} / \partial \tau>0$, the higher the average price of tourism products is, the higher the reward and punishment intensity. The certificate is completed.

According to theorem 1, in order to maximize the utility of tourists, travel agencies should reduce the intensity of rewards and punishments to maximize their own interests if they give tour guide service providers a higher share of performance. Due to the invisibility of tour guide services and the heterogeneity of service standards, there will be certain errors in the supervision and observation of guide service providers by travel agencies. When the error is too large, it will inevitably affect the immediate interests of guide service providers and the continuation of their cooperation. In order to maintain the interests of all parties under the condition 
of high observation errors, travel agencies have to reduce the intensity of rewards and punishments to weaken the influence of the observation errors on tour guide service providers. The increase of the external uncertainty of the tourism market will have a great restriction on the tour guide service. Setting a higher reward and punishment intensity is a necessary means to restrain the guide service providers. However, in the face of guide service providers with a high degree of risk aversion, travel agencies often set too high reward and punishment intensity will bring more insecurity to such guide service providers and restrict their work enthusiasm. When the expected travel experience of tourists increases with the increase of the average price of tourism products, in order to ensure the satisfaction of tourists, travel agencies will inevitably increase the intensity of rewards and punishments to restrain the behavior of guide service providers.

\section{(2) Analysis of performance sharing coefficient}

Theorem 2: Regardless of whether rewards and punishments are set or not, the performance-sharing coefficient is a decreasing function of the degree of risk aversion of guide service providers, and increases with the degree of external uncertainty in the tourism market.

After setting the rewards and punishment, the guide service provider's performance sharing will reduce, and the greater the reward and punishment intensity, the smaller the performance sharing will be, and the difference in performance sharing coefficient between the two cases will increase. Performance sharing coefficient is also an increasing function of travel agency's observation error to tour guide service providers.

Before setting rewards and punishments, the performance-sharing coefficient is an increasing function of the average pricing of tourism products. After setting rewards and punishments, the performance-sharing coefficient is a decreasing function of the average pricing of tourism products.

Proof: The simultaneous equations (19) and (20) can further describe the performance-sharing coefficient under the reward and punishment mechanism as:

$$
\beta^{* *}=\frac{c_{A} \tau^{2} k_{G}^{3} \rho \delta^{2}}{\tau^{2} k_{G} k_{A}^{3}+c_{A} k_{G} \rho \sigma^{2}+\rho \delta^{2}\left(c_{A} \tau^{2} k_{G}^{3}+c_{G} \tau^{2} k_{A}^{3}+c_{A} c_{G} \rho \sigma^{2}\right)}
$$

According to (11) and (22), respectively, the partial derivation of $\beta^{*}$ and $\beta^{* *}$ with respect to $\rho$ is obtained $\partial \beta^{*} / \partial \rho<0$ and $\partial \beta^{* *} / \partial \rho<0$, that is, regardless of whether the reward and punishment mechanism is set or not, the performance sharing coefficient increases with the decrease of the risk avoidance degree of the tour guide service provider.

For $\beta^{*}$ and $\beta^{* *}$, we can obtain the partial guides for $\sigma^{2}$ as $\partial \beta^{*} / \partial \sigma^{2}>0$, $\partial \beta^{* *} / \partial \sigma^{2}>0$, respectively, indicating that the higher the degree of external uncertainty in the tourism market, the lower the performance sharing coefficient. 
Then we ask $\beta^{* *}$ about the partial guidance of $\delta^{2}$, obtained $\partial \beta^{* *} / \partial \delta^{2}>0$, which indicates that the performance sharing coefficient is an increasing function of the travel agency's observation error to the tour guide service provider. Finally, the partial guides for $\beta^{*}$ and $\beta^{* *}$ to $\tau$,obtained $\partial \beta^{*} / \partial \tau>0$, which means before the reward and punishment mechanism is set, the performance-sharing coefficient is an increasing function of the average pricing of tourism products.; and $\partial \beta^{* *} / \partial \tau<0$ which means after the reward and punishment mechanism is set, the performancesharing coefficient is an decreasing function of the average pricing of the tourist product.

Then, by equation (20), the partial derivative of $\beta^{* *}$ with respect to $\gamma$ can be obtained $\partial \beta^{* *} / \partial \gamma<0$, that is, the greater the coefficient of reward and punishment, the lower the performance sharing coefficient. Recombining equations (11) and (20) shows the difference between $\beta^{*}$ and $\beta^{* *} \Delta \beta=\beta^{*}-\beta^{* *}=\frac{c_{A} \gamma \tau k_{G}^{2}}{c_{A} \tau^{2} k_{G}^{3}+c_{G} \tau^{2} k_{A}^{3}+c_{A} c_{G} \rho \sigma^{2}}>0$, that is, $\beta^{*}>\beta^{* *}$. Then, obtain the partial derivative of $\gamma$ for $\Delta \beta$ $\partial(\Delta \beta) / \partial \gamma=\partial\left(\beta^{*}-\beta^{* *}\right) / \partial \gamma>0$.We can see that the difference between the performance sharing coefficients in the two cases with/without reward and punishment mechanism increases with the increase of the reward and punishment intensity.

Combined with theorem 1 , the coefficient of reward and punishment intensity decreases with the increase of the performance-sharing coefficient, that is, there is complementarity between the two. The certificate is completed.

According to Theorem 2, in the case of the presence/absence of reward and punishment mechanisms, the degree of uncertainty in the tourism market increases, which tends to bring risks to the guide service providers and restrict their enthusiasm for work. At this time, it is necessary and effective for travel agencies to increase the performance-sharing coefficient to motivate them to be responsible and to serve. Of course, tour guide providers with a high degree of risk regulation are often unable to take on more external risks and internal responsibilities, and their performance sharing will decrease accordingly.

After setting up the reward and punishment mechanism, combining theorem 1 and theorem 2, when the travel agency's observation error of the tour guide service provider increases, the reward and punishment intensity will lower accordingly, and the reward and punishment degree coefficient and the performance sharing coefficient are negatively correlated, then the performance sharing of service providers will increase.

Moreover, the performance-sharing coefficient decreases with the setting of the reward and punishment mechanism. The difference of the performance-sharing coefficient indicates that after setting up the reward and punishment mechanism, the travel agency will inevitably transfer the original part of the performance sharing 
into the reward and punishment form to the guide service providers in order to guarantee its own income. All the above illustrate the complementarity between the reward and punishment mechanism and the performance sharing mechanism.

(3) Analysis of optimal effort level

Theorem 3: Regardless of whether the reward and punishment mechanism is set, the effort level of the tour guide service provider increases with the increase of the performance-sharing coefficient, and is a reduction function about the degree of its own risk aversion and the degree of external uncertainty of the tourism market. When the reward and punishment mechanism is set, the level of effort of the guide service provider decreases with the increase of the reward and punishment intensity. In addition, the increase of the observation error of the guide service provider will also lead to the decrease of the effort level.

Proof: Based on the formula (9), obtain the relative derivative $e_{G}^{*}$ of $\beta$ $\partial e_{G}^{*} / \partial \beta>0$, and from the simultaneous (17) and (20) obtain and the partial derivative $e_{G}^{* *}$ of $\beta \frac{\partial e_{G}^{* *}}{\partial \beta}=\frac{\tau k_{G}^{2}}{c_{G}}-\frac{\tau k_{G}^{3}}{c_{G}\left(k_{G}+c_{G} \rho \delta^{2}\right)}$. It is easy to prove that $\partial e_{G}^{* *} / \partial \beta>0$, in summary, the level of effort of the guide service provider is the increase function of the performance-sharing coefficient. Further, in conjunction with (17) and (20), the partial derivative $e_{G}^{* * *}$ of $\gamma \frac{\partial e_{G}^{* *}}{\partial \gamma}=\frac{\tau k_{G}}{c_{G}}-\frac{k_{G}+c_{G} \rho \delta^{2}}{c_{G}} . \partial e_{G}^{* *} / \partial \gamma<0$, which indicates that the level of effort of the tour guide service provider is a function of decreasing the coefficient of reward and punishment.

From the simultaneous equations (9) and (11), (17) and (19), we can obtain equations (23) and (24):

$$
\begin{aligned}
& e_{G}^{*}=\frac{c_{A} \tau^{3} k_{G}^{5}}{c_{G}\left(c_{A} \tau^{2} k_{G}^{3}+c_{G} \tau^{2} k_{A}^{3}+c_{A} c_{G} \rho \sigma^{2}\right)} \\
& e_{G}^{* * *}=\frac{c_{A} \tau^{3} k_{G}^{5} \rho \delta^{2}+\tau^{2} k_{G}^{3} k_{A}^{3}+c_{A} \tau k_{A}^{3} \rho \sigma^{2}}{c_{G}\left[\tau^{2} k_{G} k_{A}^{3}+c_{A} k_{G} \rho \sigma^{2}+\rho \delta^{2}\left(c_{A} \tau^{2} k_{G}^{3}+c_{G} \tau^{2} k_{A}^{3}+c_{A} c_{G} \rho \sigma^{2}\right)\right]}
\end{aligned}
$$

According to the equations (23) and (24), we can obtain the partial $e_{G}^{*}$ and $e_{G}^{* *}$ for the partial $\rho$ and $\sigma^{2}$ derivatives respectively, and obtain the sum $\partial e_{G}^{*} / \partial \rho<0$ and $\partial e_{G}^{* *} / \partial \rho<0$, and the sum $\partial e_{G}^{*} / \partial \sigma^{2}<0$ and $\partial e_{G}^{* *} / \partial \sigma^{2}<0$ respectively in sequence. This shows that in the case of the presence/absence of reward and punishment mechanisms, the level of effort of guide service providers is a reduction function of the degree of risk aversion and the degree of external uncertainty of the tourism market. According to equation (24), we obtain the partial $e_{G}^{* *}$ derivative of $\delta^{2} \partial e_{G}^{* *} / \partial \delta^{2}<0$, that is, the level of the tour guide service effort decreases as the travel agency increases its observation error. 
According to Theorem 3, the benefits of guide service providers increase with the increase of the performance-sharing coefficient, and the level of effort increases. This indicates that the performance sharing mechanism has a positive and effective incentive for guide service providers.

In combination with theorem 2 , tour guide service providers with a high degree of risk aversion usually have no intention to take too many risks and responsibilities, and their corresponding performance sharing will be reduced, which will lead to a reduction in their efforts. When the degree of uncertainty in the tourism market increases, those guide service providers with a high degree of risk aversion will reduce the level of effort because they cannot avoid this effect. When the travel agency sets up the reward and punishment mechanism, the level of effort of the tour guide service provider has not been improved due to the mandatory constraint of the reward and punishment mechanism, but has decreased; and the greater the coefficient of reward and punishment, the lower the level of effort of the tour guide service provider. This means that the reward and punishment mechanism failed to exert its effective incentive effect, but brings negative influence to the guide service providers.

The increase in the coefficient of reward and punishment will lead to a decrease in the performance sharing of guide service providers, and the original incentive effect of the performance sharing mechanism will be weaker. Therefore, it is crucial for travel agencies to set the intensity of rewards and punishments reasonably.

Theorem 4: The effort level of travel agencies is significantly increased with the setting of reward and punishment mechanism, and the greater the reward and punishment intensity coefficient, the higher the effort level of travel agencies, that is, the reward and punishment mechanism has a hidden incentive effect on travel agencies. The level of effort of travel agencies is a decreasing function of performance sharing coefficient, risk aversion degree of guide service providers and observation error of guide service providers by travel agencies, and an increasing function of external uncertainty degree of tourism market.

Proof: According to formula (10) and formula (18), obtain $e_{A}^{*} 、 e_{A}^{* * *}$ and about $\beta$ $\partial e_{A}^{*} / \partial \beta<0$ and $\partial e_{A}^{* *} / \partial \beta<0$ respectively, that is, the effort level of travel agency decreases with the increase of performance sharing coefficient. In combination with the conclusion in theorem 2, $\beta^{*>} \beta^{* *}$, we can obtain that $e_{A}^{* *}-e_{A}^{*}=\left(\beta^{*}-\beta^{* *}\right) \tau k_{A}^{2} / c_{A}>0, e_{A}^{* *}>e_{A}^{*}$, which means the effort level of travel agencies after setting reward and punishment mechanism is greater than that of tour guide service providers without considering reward and punishment. Combining equations (18) and (20) $e_{A}^{* * *}=\frac{\gamma \tau k_{A}^{2}\left(k_{G}+c_{G} \rho \delta^{2}\right)}{c_{A} \tau k_{G}^{2}}$, we can find the partial guidance $e_{A}^{* *}$ of $\gamma, \partial e_{A}^{* * *} / \partial \gamma>0$, that is, the travel agency's own effort level increases with the increase of the reward and punishment intensity to the tour guide service provider. 
Recombining equations (18) and (19), we can obtain equations (26):

$$
e_{A}^{* *}=\frac{\tau^{3} k_{G} k_{A}^{5}+c_{A} \tau k_{G} k_{A}^{2} \rho \sigma^{2}+\rho \delta^{2}\left(c_{G} \tau^{2} k_{A}^{3}+c_{A} c_{G} \rho \sigma^{2}\right)}{c_{A}\left[\tau^{2} k_{G} k_{A}^{3}+c_{A} k_{G} \rho \sigma^{2}+\rho \delta^{2}\left(c_{A} \tau^{2} k_{G}^{3}+c_{G} \tau^{2} k_{A}^{3}+c_{A} c_{G} \rho \sigma^{2}\right)\right]}
$$

We can seek the partial deviation $e_{A}^{* *}$ of $\sigma^{2} 、 \rho$ and $\delta^{2}$ in turn. The result is $\partial e_{A}^{* *} / \partial \sigma^{2}>0$, that is, the travel agency's level of effort increases with the increase of the degree of external uncertainty in the tourism market; $\partial e_{A}^{* * *} / \partial \rho<0$ and $\partial e_{A}^{* *} / \partial \delta^{2}<0$, that is, the greater the risk avoidance degree of tour guide service providers and the greater the observation error of travel agencies, the smaller the effort level of travel agencies.

Based on theorem 4, when the reward and punishment mechanism is set, the performance sharing of the guide service provider will decrease, and the performance sharing obtained by the travel agency will increase, and the level of the travel agency's efforts will increase accordingly.

According to theorem 1 and theorem 2, with the increase of the reward and punishment intensity coefficient, the performance sharing of corresponding tour guide service providers will decrease, which means that the travel agency will get more performance sharing, and its effort level will also increase. On the other hand, from a deeper level, although travel agencies have set up reward and punishment mechanism for guide service providers, they have also obtained implicit and potential incentives. In addition, travel agencies' observation error on tour guide service providers is too large, which improves the performance sharing of tour guide service providers, resulting in a decrease in the performance sharing of travel agencies, thus affecting their efforts.

When the degree of uncertainty in the tourism market becomes greater, guide service providers will reduce their effort. In order to maintain the system output of guide service outsourcing, travel agencies will have to improve their own efforts. However, when a guide service provider cooperating with the travel agency with low risk aversion, it will affect the enthusiasm of the travel agency negatively, resulting in reduction of their efforts.

(4) Analysis of tourists' utility

Under the dual agency relationship, the effectiveness of visitors will directly depend on the level of effort of travel agencies and guide service providers. According to the above analysis, the two levels of effort are directly subject to performance sharing and reward and punishment intensity. That is to say, the performance-sharing coefficient and the incentive coefficient of the travel agency affects the utility of tourists indirectly. The following will analyze the impact of the performance-sharing coefficient and the incentive coefficient on the utility of visitors. 
According to formulas (1), (9) and (10), we can obtain the function of the utility and performance-sharing coefficient of the non-reward and punishment mechanism (27), combined with formulas (1), (17) and (18), we can obtain the function of the tourist utility and the performance sharing coefficient of the reward and punishment mechanism. (28). Combined with the formulas (28) and (20), we can obtain the relationship between the utility of the visitor and the intensity coefficient of the reward and punishment (29).

$$
\begin{gathered}
\Pi_{T}^{*}=v+\frac{\tau k_{A}^{3}(1-\beta)(\theta-\tau)}{c_{A}}+\frac{\beta \tau k_{G}^{3}(\theta-\tau)}{c_{G}} \\
\Pi_{T}^{* *}=v+\frac{\tau k_{A}^{3}(1-\beta)(\theta-\tau)}{c_{A}}+\frac{\beta \tau k_{G}^{3}(\theta-\tau)}{c_{G}}+\frac{\tau k_{G}^{4}(1-\beta)(\theta-\tau)}{c_{G}\left(k_{G}+c_{G} \rho \delta^{2}\right)} \\
\Pi_{T}^{* *}=v+\frac{\tau k_{G}^{3}(\theta-\tau)}{c_{G}}+\frac{k_{G}^{2}(\theta-\tau) \gamma}{c_{G}}+\frac{k_{A}^{3}(\theta-\tau)\left(k_{G}+c_{G} \rho \delta^{2}\right) \gamma}{c_{A} k_{G}^{2}} \\
-\frac{(\theta-\tau)\left(k_{G}+c_{G} \rho \delta^{2}\right) \gamma}{c_{G}}
\end{gathered}
$$

In order to more intuitively present the relationship between the tourist utility and the performance sharing coefficient and the reward and punishment intensity coefficient, the specific parameters are given below and the MATLAB numerical simulation is performed.

Table. 1: Parameters of tour guide service outsourcing service contract under dual principal-agent relationship

\begin{tabular}{|c|c|c|c|c|c|c|c|c|c|c|c|}
\hline Parameters & $C_{G}$ & $C_{A}$ & $K_{G}$ & $K_{A}$ & $\tau$ & $\delta^{2}$ & $\gamma$ & $\theta$ & $v$ & $\beta$ & $\gamma$ \\
\hline Values & 0.3 & 0.4 & 0.2 & 1.3 & 1.0 & 4.0 & 1.2 & 1.5 & 2.0 & 0.2 & $\ldots$ \\
& & & & & & & & & & $\ldots 1.0$ & 0.0 \\
\hline
\end{tabular}

According to Figure 2, in the absence of a reward and punishment mechanism, the tourist utility increases with the increase of the performance sharing-coefficient, and the increase is relatively slow.

Combined with theorem 3, when the performance sharing coefficient increases, the level of effort of the guide service provider will inevitably increase. At this time, the guide service provider will actively bring a high tourist experience to the tourists, thus improving the utility of the tourists. However, subject to the impact of performance analysis on the level of travel agency efforts (according to Theorem 4), the increase in visitor utility with the performance-sharing factor is small. When the 
reward and punishment mechanism is set, the utility of the visitor will obviously be greater than the effect when the reward and punishment mechanism is not set, and will decrease as the performance sharing coefficient increases.

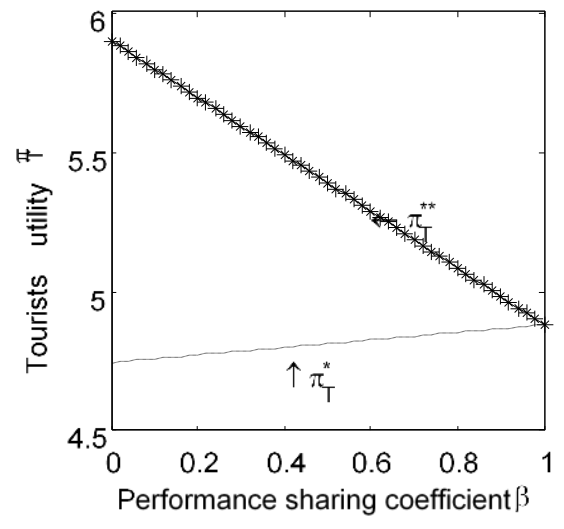

Figure 2. $\beta$ sensitivity analysis

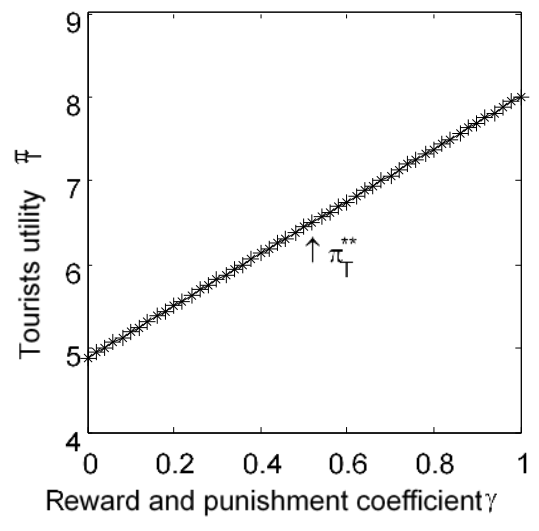

Figure 3. $\gamma$ sensitivity analysis

The direct cause of the decrease in the performance-sharing coefficient of the tourists is that after the reward and punishment mechanism is set, the performance sharing of the guide service providers is reduced and the rewards and punishments revenues are uncertain. These affect the level of their efforts, thus indirectly restricting the effectiveness of tourists and make the passenger utility curve slope downwards.

As can be seen from Figure 3, the utility of tourists always increases with the increase of the intensity coefficient of rewards and punishments. According to Theorem 3, when the coefficient of reward and punishment increases, the level of effort of the guide service provider will decrease, but the utility of the visitor has not decreased. This is because after the increase of the coefficient of reward and punishment, the performance sharing of travel agencies will gradually increase and stimulate their work enthusiasm, prompting travel agencies to play a key role in the outsourcing process. However, from the perspective of successful implementation of guide service outsourcing, travel agencies should appropriately set the intensity of rewards and punishments while ensuring their own interests. This will not only help maintain the normal and consistent operation of the tour guide service outsourcing system, ensure the tourist experience, but also promote a harmonious, trusting and sustainable partnership between the travel agency and the guide service provider.

\section{Conclusion}

The travel agency-single guide service provider has expanded into a dual agency relationship among tourists, travel agencies and guide service providers. The travel 
agency acted as a dual principal-agent and established a cooperative service relationship with guide service providers.

Based on the above principal-agent relationship, we construct the cooperative service outsourcing contract under the two situations of no reward and punishment mechanism and reward and punishment mechanism respectively, and study the relevant incentive parameters and the effectiveness of tourists separately.

Through model analysis and numerical simulation, we can find that the addition of reward and punishment mechanism based on the performance sharing mechanism can effectively improve the effectiveness and satisfaction of tourists as well. In terms of incentive effectiveness, the performance sharing mechanism and the reward and punishment mechanism are complementary to each other. However, the reward and punishment mechanism has not improved the level of efforts of guide service providers but has reduced them.

Through numerical simulations, we can find that although the level of efforts of guide service providers decreases, it has not caused a decrease in the effectiveness of tourists. This means that travel agencies play an important role in maintaining and improving the effectiveness of tourists. However, the higher coefficient of reward and punishment can only ensure the effectiveness of tourists and improve the income of the travel agency. It may damage the interests of tour guides and is not enough to maintain long-term and harmonious partners of travel agencies and tour guides in guide service outsourcing.

Reasonable setting of performance sharing mechanism and reward and punishment mechanism is extremely important for travel agencies to choose the guide service outsourcing strategy.

(The paper is funded by Hunan Natural Science Foundation(2018JJ3131))

\section{References}

Athena, H. M., Kevin, K. W. \& Richard, C. C. (2011) Critical issues affecting the service quality and professionalism of the tour guides in Hong Kong and Macau. Tourism Management, 32(6): 1442-1452.

Athena, H. M., Kevin, K. W. \& Richard, C. C. (2010). Factors affecting the service quality of the tour guiding profession in Macau. International Journal of Tourism Research, 12(3): 205-218.

Atilgan, E., Akinci, S. \& Aksoy, S. (2003). Mapping service quality in the tourism industry. Managing Service Quality, 13(5): 412-422.

Bei, F. \& Caiyun, T. (2012). Study on theoretical building for tourism service outsourcing. Tourism Science, 26(2): 29-39. 
Cezar, A. \& Cavusoglu, H., Raghunathan S. (2014). Outsourcing information security: Contracting issues and security implications. Management Science, 60( 3$): 638-657$.

Chatzoglou, P. \& Sarigiannidis, L . (2009). Business outsourcing and organizational performance: the case of the Greek hotel industry. International Journal of Services Technology and Management, 11(2):105-127.

Delreux, T. \& Adriaensen, J. (2017). Twenty years of principal-agent research in EU politics : how to cope with complexity?. European Political Science, (3): 1-18.

Flemming, S. \& Jens, F. J. (2015). Value creation and knowledge development in tourism experience encounters. Tourism Management, 46:336-346.

Gina, I. B., Mirela, S. \& George, M. M. (2014). Alternative method of quality evaluation in tourism. Case study applied in tourist accommodation units. Procedia Economics and Finance, 15:671-678.

Gronroos, C. (1982). Strategie management and marketing in service sector. MA: Marketing Science Institute.

Haiyan, S., Shu, Y. \& George, H. Q. (2009). Price interactions between theme park and tour operator. Tourism Economics, 15(4): 813-824.

Holmstrom, B. \& Milgrom, P. (1991). Multi-task principal agent problems: Incentive contracts, asset ownership, and job design. Journal of Law, Economics and Organization, 7: 24-52.

Kristensen, S. R., Siciliani, L. \& Sutton, M. (2016). Optimal price-setting in pay for performance schemes in health care . Journal of Economic Behavior \& Organization, 123: 57-77.

Lamminmaki D. (2008). Accounting and the management of outsourcing: An empirical study in the hotel industry - Management Accounting Research, 19(2):163-181.

Roberto R. M. C. (2006). Tourism service quality begins at the airport. Tourism Management, 27(5):874-877.

Rud, O. A., Rabanal, J. P. \& Horowitz, J. (2017). Does Competition Aggravate Moral Hazard? A Multi-Principal-Agent Experiment . Journal of Financial Intermediation.

Simon, H., Paul, H. \& Graham, A.M. (2004). The Measurement of service quality in the tour operating Sector: a methodological comparison. Journal of Travel Research, 42(3): 305-312. 
Susarla, A., Subramanyam, R. \& Karhade, P. (2010). Contractual provisions to mitigate holdup: evidence from information technology outsourcing. Information Systems Research, 21(1):37-55.

Walden, E. A., Hoffman, J. J. (2007). Organizational form, incentives and the management of information technology: Opening the black box of outsourcing. Computers \& Operations Research, 34(12): 3575-3591.

Wu, D. J., Ding, M. \& Hitt, L. M. (2013). IT implementation contract design: Analytical and experimental investigation of IT value, learning, and contract structure. Information Systems Research, 24(3) :787-801. 